\title{
BMJ Open Cohort profile: The Swiss Transplant Cohort Study (STCS): A nationwide longitudinal cohort study of all solid organ recipients in Switzerland
}

Susanne Stampf (1) , ${ }^{1}$ Nicolas J Mueller, ${ }^{2}$ Christian van Delden, ${ }^{3}$ Manuel Pascual, ${ }^{4}$ Oriol Manuel (1) , ${ }^{4,5}$ Vanessa Banz, ${ }^{6}$ Isabelle Binet, ${ }^{7}$ Sabina De Geest, ${ }^{8}$ Pierre-Yves Bochud, ${ }^{5}$ Alexander Leichtle (D) , ${ }^{9}$ Stefan Schaub, ${ }^{1}$ Jürg Steiger, ${ }^{1}$ Michael Koller, ${ }^{1}$ Swiss Transplant Cohort Study

To cite: Stampf S,

Mueller NJ, van Delden C, et al. Cohort profile: The

Swiss Transplant Cohort Study (STCS): A nationwide longitudinal cohort study of all solid organ recipients in Switzerland. BMJ Open 2021;11:e051176. doi:10.1136/ bmjopen-2021-051176

- Prepublication history for this paper is available online. To view these files, please visit the journal online (http://dx.doi. org/10.1136/bmjopen-2021051176).

Received 24 March 2021 Accepted 11 November 2021

Check for updates

(C) Author(s) (or their employer(s)) 2021. Re-use permitted under CC BY-NC. No commercial re-use. See rights and permissions. Published by BMJ.

For numbered affiliations see end of article.

Correspondence to Dr Susanne Stampf; susanne.stampf@usb.ch

\section{ABSTRACT}

Purpose The Swiss Transplant Cohort Study (STCS) is a prospective multicentre cohort study which started to actively enrol study participants in May 2008. It takes advantage of combining data from all transplant programmes in one unique system to perform comprehensive nationwide reporting and to promote translational and clinical post-transplant outcome research in the framework of Swiss transplantation medicine. Participants Over 5500 solid organ transplant recipients have been enrolled in all six Swiss transplant centres by end of 2019, around three-quarter of them for kidney and liver transplants. Ninety-three per cent of all transplanted recipients have consented to study participation, almost all of them (99\%) contributed to bio-sampling. The STCS genomic data set includes around 3000 patients.

Findings to date Detailed clinical and laboratory data in high granularity as well as patient-reported outcomes from transplant recipients and activities in Switzerland are available in the last decade. Interdisciplinary contributions in diverse fields of transplantation medicine such as infectious diseases, genomics, oncology, immunology and psychosocial science have resulted in approximately 70 scientific papers getting published in peer-review journals so far.

Future plans The STCS will deepen its efforts in personalised medicine and digital epidemiology, and will also focus on allocation research and the use of causal inference methods to make complex matters in transplant medicine more understandable and transparent.

\section{INTRODUCTION}

Starting with the first kidney transplantation (TX) in 1964 in Switzerland, the University Hospitals of Bern, Geneva, Lausanne and Zurich as well as the Cantonal Hospital of St. Gallen are certified to perform solid organ $\mathrm{TX}^{1}{ }^{1}$ The six TX centres ensure high-quality TX care according to international standards. In early years they had worked largely independently of each other, mainly following regulations of the federal states. In 2007, the
Strengths and limitations of this study

- The Swiss Transplant Cohort Study (STCS) is a prospective, observational study enrolling all solid organ transplant recipients in Switzerland since May 2008.

- The STCS has a scalable data infrastructure that maps the complexity of post-transplant patient care and allows identifying new pathways contributing to the understanding of patient and allograft outcome.

- The STCS has established a thorough fundament to reflect the multifaceted nature of solid organ transplantation processes, to assess determinants of short-term and long-term post-transplant outcome, to understand and support decision-making processes of post-transplant patient healthcare, and to record and update risk profiles to report changes in disease and treatment status.

- Data contents include diverse areas such as clinical data, patient-reported outcome data, bio-banking and genomic data, specifically adapted for the needs of personalised transplantation care.

- STCS biobank-sampling from consenting transplant recipients is performed at three fixed time points within the first year after transplantation, but not beyond or at relevant disease-specific events such as rejection or infection.

Federal Act on Transplantation came into force regulating the TX of organs, tissues and cells and establishes the political guideline of a nationwide reporting throughout Switzerland. ${ }^{2}$ In 2006, the Swiss Transplant Cohort Study (STCS) was founded as a nationwide, open, prospective, multicentre cohort study of all solid organ transplant (SOT) recipients in Switzerland. First patients were enrolled in May 2008. ${ }^{34}$ The aim of the STCS has always been twofold. First, in accordance with legal regulations and requirements of the Federal Office of Public Health (FOPH), the STCS 
performs the systematic monitoring of minimal compulsory information about SOT recipients lifelong post TX. The responsibility for the legally required public reporting of transplant outcomes results in annual reports issued by the STCS for the public, various stakeholders and the FOPH. ${ }^{5}$ Second, the STCS fosters an efficient collaboration between all Swiss TX centres, allowing for harmonisation and standardisation of data acquisition with regard to the TX centres' activities and enabling comprehensive observational and translational research in TX medicine and its specialties. ${ }^{3}$ Therefore, the STCS collects broad information on transplant recipients, transplanted organs and the TX process itself, and prospectively observes key long-term post-transplant outcomes in high granularity. To consolidate and manage all information, the STCS offers an elaborate data infrastructure to successfully pursue its objectives.

In Switzerland, several stakeholders and institutions constitute the transplant sector and operate in a tightly integrated network. On behalf of the FOPH, the foundation Swisstransplant operates as an independent organisation for donor recruitment and organ procurement in Switzerland. ${ }^{1}$ It also manages organs that are offered or requested from abroad. ${ }^{6}$ The Swiss Organ Allocation System (SOAS) is a web-based system provided by the FOPH and used by Swisstransplant to centrally allocate organs by an algorithm prioritising candidates according to their time on the waitlist and their risk of dying on it, as well as according to the immunological constellation between donor and candidate. ${ }^{7-9}$ As already established in other countries and first carried out in Switzerland in 1999, an official programme for kidney paired donations was launched in 2019. ${ }^{11011}$ It enables kidney swaps among transplant candidates with incompatible living donors (from relatives or partners) and has been regulated as an statutory ordinance in the Swiss Transplantation Act since November 2017. ${ }^{12}$ In 1993, the first register of living donor organs (SOL-DHR) was established in Switzerland with the purpose to provide transparency about donor origin, and to capture short and long-term consequences of living kidney and liver donors. ${ }^{13} 14$ In line with the SOLDHR, the STCS provides an extensive pool of high-quality data of SOT recipients.

\section{COHORT DESCRIPTION}

\section{STCS organisation}

Since its initiation, the STCS has a well-established organisational structure. ${ }^{14}$ It is operationally led by an Executive Office (EO), the STCS steering committee, called board of representatives, which acts as governing-body and includes representatives of all TX centres. The STCS scientific committee, comprised representatives from all participating centres and various medical specialties and is responsible for all aspects regarding the conduct of STCS nested projects (NPs). The NPs represent the central element of scientific operation and productivity within the STCS framework. To date, more than
150 STCS NPs have been approved. The STCS Data Centre is the operator of the data infrastructure and all derived data processes. It performs all data management processes including the maintenance of the IT environment. Data centre services involve assisting in data preparation and in obtaining third party data for record linkage or in the collection of project-specific ('add-on') data. STCS working groups provide subject expertise for data definitions and collection, and promote research in their subject areas. They ensure compliance with international guidelines. ${ }^{15-18}$ The STCS 'Psychosocial Interest Group' (PSIG) is in charge of managing and updating of the STCS psychosocial questionnaire. ${ }^{19-22}$ The STCS 'Infectious Diseases' (ID) working group maintains a thorough ID dataset based on definitions in accordance with international standards. This has enabled valuable contributions to international collaborations. ${ }^{23-26}$ The STCS 'Immunology' working group provides detailed immunological test data and validates the donor-specific HLA antibody (DSA) assignment. ${ }^{27-29}$ Development of post-transplant solid organ, especially post-transplant lymphoproliferative disorders (PTLD), and skin cancer is the focus of the STCS 'Oncology' working group. ${ }^{30-32}$ The recently launched STCS 'Paediatric' group meets the needs of paediatric transplant recipients. The STCS 'Genetics' working group manages access and use of genetic data from more than 3000 SOT recipients. The genomic dataset allows for identification of single nucleotide polymorphism associated with a potentially wide variety of transplant-related phenotypes either by a candidate-gene ${ }^{33-35}$ or by a genome-wide approach. ${ }^{36}$ The precise description of multiple phenotypes linked with a comprehensive prospective collection of pertinent endpoints underpins the value of the STCS. ${ }^{37}$ The STCS 'Lab' working group organises the decentralised, centre-based biobank in which samples collected from all consenting patients are managed. All activities of the biobank are in line with the data governance structure of the STCS. Since 2019 the STCS collaborates with the Swiss Biobanking platform coordinating essentially Swiss biobanks and supporting them to align with standards. ${ }^{38}$

\section{Data governance and regulatory aspects}

The local ethics committees responsible for all six Swiss TX centres approved the STCS prior to its initiation in 2008. Since 2014, human research has been regulated in Switzerland through the Swiss legislation on human research (Federal Act on Research involving Humans, HRA and its ordinance, HRO). The STCS fully complies with the Swiss legislation as well as with the declaration of Helsinki. Moreover, as required by the Swiss Transplantation Act, publicly available STCS annual reports comprehensively reflect the quality of care post-transplant. ${ }^{5}$ Informed consent procedures are implemented since the beginning of the cohort. SOT recipients consent to STCS participation at enrolment but they can revise their participation any time later on. The STCS' informed consent broadly covers the collection and reuse of health-related personal 
and genomic data as well as the sampling of biological material for research. It also includes the linkage of STCS data and samples with data from third parties. In addition, each NP using STCS data or samples is subject to a project-specific ethics committee review and approval, and the principal investigator is responsible for ensuring compliance during project implementation. STCS data and the use of biosamples are open to researchers with an approved NP and approved sample use. The conditions for data access are stated in the guidelines for researchers and involve approval of each project by the STCS scientific committee and a competent ethics committee.

\section{Data infrastructure}

The data are conceptually divided into five distinct components: (1) non-genetic health-related personal data from transplant recipients, donor-recipient matching data and donor-derived allograft data, (2) genomic data from recipients, (3) biological material of recipients, (4) 'add-on' data and (5) third-party data from external sources. Third-party data may originate from a variety of distinct sources such as social insurance data, electronic health record data from hospitals or associated registries (eg, Swiss register for living donor organs and Swiss dialysis register). The STCS electronic codebook provides a complete metadata catalogue of all variables and concepts as a web-based query interface including the version history of changes.

The STCS maintains a professional web-based data capture system for the management of the health-related data and the administration of the biobank. The system has an inherent atomic structure composed of four principle and interconnected layers: the patient, the TX procedure, the organ and the donor. A patient may have several consecutive TXs, several organs may be transplanted simultaneously as part of one TX. A donor can donate to several recipients. In a combined TX, all organs originate from the same donor. This data structure allows to unambiguously connect detailed pretransplant and peritransplant clinical and laboratory data as well as wideranging post-transplant outcomes to SOT recipients and allografts. ${ }^{3}$ The multilayered data system also facilitates the integration of donor-recipient matching and donorderived allograft data, and genomic data, information of biological material (biobank) and external third-party data (eg, waitlist data). For NPs, small 'add-on' databases can be linked to the STCS to enrich, for example, routine clinical data from hospitals or to prospectively collect new data. ${ }^{21}$ Samples from SOT recipients are registered in the STCS database, however, they are physically stored and maintained in the local biobanks of the TX centres. A prerequisite for the release of biobank samples is a sample-ordering list generated and verified by the STCS Data Centre and the head of the STCS biobank providing guidelines and support to researchers. Standardised operating procedures are used to ensure sample quality and interoperability among all local STCS biobanks. Given the signed material transfer agreement, the material can be retrieved from local STCS biobanks under predefined preanalytical conditions and dispatched to the principal investigator. Remaining unused sample stocks will be stored in the on-site laboratories after the end of an NP.

The STCS considers all data as sensitive and therefore enforces the use of a highly secured tenant hosted with the BioMedIT network of the Swiss Personalised Health Network (SPHN). Between both, a service level agreement is in place to prevent non-authorised access to the data at the tenant. Among other things, only pseudonymised data are stored and used for research purposes.

\section{Study design}

The STCS as a nationwide multicentre ongoing cohort study was set-up with a time fixed follow-up schedule at the time of TX, six and twelve-month post TX and yearly after, STCS enrolment is defined as the patient's baseline. If any further TX is performed, additional assessments along the new TX at 6 and 12 months are conducted with data updates at these time points. After completion of these three extra visits, the future follow-up visit schedule is synchronised with the patient's first STCS TX. If SOT recipients refuse or withdraw their consent, a mandatory set of minimal data is gathered by law. ${ }^{2}$ It essentially consists of recipients' demographic data necessary for allograft compatibility, for example, blood group and HLA phenotyping. Furthermore, relevant posttransplant clinical events affecting allograft survival and recipient's vital status are also recorded as minimal data. For recipients providing written consent to STCS, the full research dataset is collected including biobank samples and patient-reported outcomes. ${ }^{3}$ The STCS psychosocial questionnaire is completed at 6 months, $1,3,5$, and 10 years after TX and then every 5 years, starting with admission on the waiting list.

\section{Data acquisition}

Standard patient and allograft-specific evaluations are carried out at each follow-up visit at the end of predefined time intervals. Information on serious patient or allograft events and possible treatment is recorded whenever they occur, such as patient death, allograft rejection or failure, or infections leading to hospitalisation. Relevant information about transplant and donor characteristics are gathered at the time of TX. The source of donor data in the STCS is the SOAS. The IT system allows for data entry at any time. Data are validated at the end of each follow-up interval and the data input is then closed for this period.

\section{Measurements}

Recipient data include demographic and routine laboratory data as well as detailed information about underlying organ diseases leading to TX. Patient's medical history of cardio-pulmonary, metabolic, endocrine or kidney diseases, cancer, major previous IDs and previous TXs is also captured. The STCS psychosocial questionnaire assesses patient-reported outcomes (eg, healthrelated quality of life (EQ-5D: EuroQOL Five Dimensions 
Questionnaire), depressive symptomatology (Hospital anxiety and depression score (HADS)), sleep quality, medication adherence, smoking) and selected sociodemographic variables (eg, educational level, professional status). A specific repository for medication data (including primarily induction, maintenance immunosuppression, ID prophylaxis drugs) complements the recipient data.

Depending on the organ in need of TX, extensive organrelated data are gathered, for example, dialysis modality in case of kidney TX, 'model for end-stage liver disease' (MELD) score, ascites, hepatic encephalopathy for liver TX, current or past mechanical ventilation, forced expiratory volume (FEV) and $6 \mathrm{~min}$ walking test in case of lung TX, and typical information as extent of failure (as 'New York Hospital Association' (NYHA) classes), ejection fraction, $\mathrm{VO} 2_{\max }$ for heart transplant recipients. Procedurerelated information is often organ-specific and includes, in addition to cold ischaemia time collected for all TX, for example, the bypass and reperfusion time in lung and heart TX and any transplant complications that may arise. Transplant-related biosamples from SOT recipients taken at the time of TX, 6 and 12 months after TX involve DNA (only at baseline), plasma and viable cells. Information concerning the number of aliquots that have been collected, are still in stock and have been already used are captured.

Both the donor and the recipient candidate undergo a series of serology assessments and HLA typing for histocompatibility, and immunological risk assessment by virtual crossmatching (eg, HLA-DSA assignment). ${ }^{39}$ Further peritransplant information on donor and recipient potential pre-existing exposure to ID such as Cytomegalovirus (CMV) and Epstein-Barr virus is gathered. General donor characteristics like age and gender and type of donation supplement the pool of donor-matching data.

\section{Outcomes}

The STCS collects and provides a variety of phenotypical post-transplant data without specifying primary or secondary outcomes. Patient and transplant-related outcomes that are frequently used comprises patient and allograft survival, occurrence of biopsy-proven rejections, clinically significant IDs, (skin) cancer and patientreported outcomes. Information on causes of death and allograft failure, type of rejection, ID and cancer, and associated consequences, for example, hospitalisation and change of medication, which describe the severity of these events, complete the outcome data. Longitudinal measurements of transplant function, for example, estimated glomerular filtration rate in kidney and FEV in lung transplants, and any other kind of patient or graft disease are also comprehensively covered.

\section{Patient and public involvement}

The STCS has embraced the new research paradigm by involving patients in the whole research cycle. A patient involvement infrastructure was developed and implemented over the past 4 years in the STCS. Research priorities from the patient's perspective were defined and the STCS patient advisory board was launched. Patients may participate as members in NP research teams and will be integrated into the STCS board of representatives. Research findings are reported in lay language on the STCS website. Patient involvement ensures that research is relevant to patient needs and provides a basis for strengthening transplant care for patients.

\section{RESULTS}

\section{Baseline data}

Between May 2008 and end of 2019, 5918 TX with 6192 organs were performed in 5672 patients, predominantly kidney, liver, lung and heart TX (figure 1). Ninety-three per cent of all enrolled SOT recipients gave written informed consent to STCS participation and almost all consenting participants contributed to biosampling $(99.8 \%)$. Even though single kidney TX are still the most frequently performed $(56.4 \%)$, the annual proportion of liver TX has increased significantly over years (from $17.7 \%$ in 2008 up to $26.8 \%$ in 2019). The proportion of rarely performed single and combined TX ('Others' in figure $1_{2}$ left panel) currently is $5 \%$, with the majority being kidney combined transplants, for example, kidneypancreas (117) and kidney-liver (51). Three different triple TX combinations were performed; a kidney-isletslung, an islets-liver-lung and a liver-pancreas-small bowel TX. Islets (54) and pancreas (23) were less frequently transplanted as single organ TX.

\section{SOT recipient enrolment}

Among 5249 consenting SOT recipients, recipient age at enrolment was similar across the four main TX programmes (kidney, liver, lung and heart) in Switzerland, with paediatric patients being more prevalent among liver and heart transplant recipients. The proportion of women was greater among lung recipients (table 1). Lung and liver transplant recipients were less likely to have multiple comorbidities. About $11 \%$ of patients already had a TX prior to STCS inclusion. Twelve per cent of all SOT recipients had a history of cancer, a quarter of them skin cancer. The proportion of former or current smokers was similar among all SOT recipients at the time of TX, even among lung transplant recipients. Inherited and endogenous disorders have led more often to heart and lung transplants, whereas alcohol consumption or tumours have predominated as cause for liver TX. Diabetes and related disorders were major causes of kidney TX.

\section{Donor-related data}

More than 3000 donors were registered within the study period. Fifty-five per cent were deceased donors, and $70 \%$ of them donated more than one organ (figure $1_{2}$ right panel). Median donor age at time of donation (54 


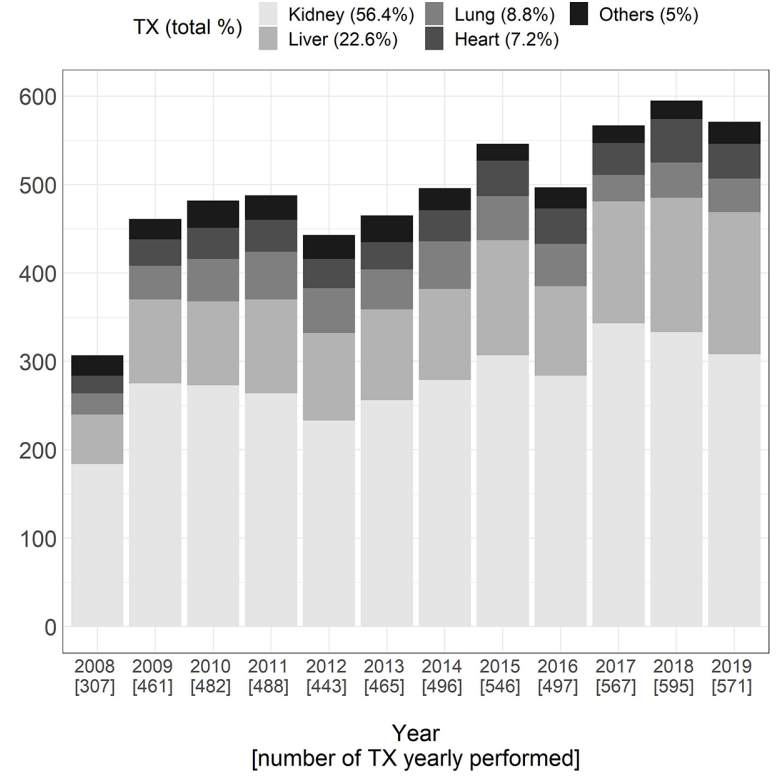

\begin{tabular}{|lr|}
\hline & N (\%) \\
\hline Number of TX performed & 5918 \\
\hline - combined TX & $218(3.7 \%)$ \\
\hline SOT recipients enrolled & 5672 \\
\hline - with consent & $5249(92.5 \%)$ \\
\hline - with history of prior TX & $549(10.5 \%)$ \\
\hline - female & $1879(35.8 \%)$ \\
\hline - age (years), Median [IQR] & $54[42,62]$ \\
\hline Donors & 3038 \\
\hline - living & $1363(44.9 \%)$ \\
\hline - deceased & $1675(55.1 \%)$ \\
\hline - DBD / DCD & $1172(70 \%)$ \\
\hline - multiple organs & $1551(51.3 \%)$ \\
\hline - female & $54[42,63]$ \\
\hline - age (years), Median [IQR] & $1487(88.8 \%) / 188(11.2 \%)$ \\
\hline
\end{tabular}

Figure 1 Number of all STCS solid organ transplantations performed between May 2008 and December 2019 (left) and basic STCS information (right). DBD, donation after brain death; DCD, donation after circulatory death; GF, graft failure; IQR, interquartile range; TX, transplantation.

years, IQR: 42-63) was similar to median recipient age at STCS enrolment, however, we recorded more female donors $(51.3 \%)$ than female recipients $(35.8 \%)$. About $40 \%$ and $5 \%$ of kidney and liver transplants, respectively, originated from living donation (table 1). Overall, only a small proportion of all deceased donations came from donors after circulatory death. A low proportion of all kidney $(6.3 \%)$ and liver TX $(0.3 \%)$, respectively, were blood group AB0 incompatible TX. A CMV high-risk constellation was more frequently observed in lung and heart transplant recipients. Pretransplant existing DSA was found in $17 \%$ of tested kidney transplant recipients.

\section{Patient and allograft survival}

Overall, the median follow-up was 4.4 years, 495 SOT recipients were followed for more than ten years, mostly after kidney TX. A total of 808 deaths were observed (15.4\%) and 64 patients were lost to follow-up (1.2\%) (table 2). Overall mortality at $1,3,5$ and 10 years after STCS enrolment was 5\%, 9.6\%, $14.4 \%$ and $25.4 \%$. The most common causes of death were graft failure (GF) and multiorgan failure, especially after lung TX where the death rate was higher than the average STCS population (36.8\% vs $15.4 \%)$. Infectious diseases led more often to death after kidney or liver TX compared with all other TX programmes. One-third of all deceased SOT recipients had a prior organ failure (figure $2_{2}$ right panel). After 10 years of follow-up, graft-failure free survival was $39 \%$ for lung, $68 \%$ for liver and $73 \%$ for heart and kidney transplant recipients (figure 32 left panel). While heart and liver failures occurred mainly shortly after TX, there was a constant high failure rate over time for lung transplants (figure 3, right panel). The likelihood of GF was initially greater than recipient death, changing after 3,4 and 6 years in heart, liver and kidney recipients.
Around $10 \%$ of all consenting SOT recipients had at least one GF during their STCS follow-up. Lung allografts failed more frequently $(17.9 \%)$ and lung failures were observed significantly later on average compared with kidney, liver and heart failures (table 2). The risk of GF was higher $(21.5 \%)$ in rare TX scenarios ('Other') often performed as combined TX, islets and pancreas in kidneypancreas TX mainly failing to function. A re-TX, that is, the same organ was transplanted again, was performed in one third of patients after GF. It was more common for liver transplant recipients (67.7\%). In total, 599 allografts failed, 543 SOT recipients had organ failure, and five of them only with regard to a second TX. (figure $2_{2}$ right panel). Eleven patients had a complete organ failure after a combined TX, that is, all organs transplanted in a combined TX failed. In contrast, 42 patients had repeated failures of allografts transplanted in different consecutive TX. Overall, 221 SOT recipients had consecutive TX after STCS enrolment, $12 \%$ of them without any GF.

The majority of SOT recipients are still alive with functioning allografts $(4110,78.3 \%)$. Around $10 \%$ of the SOT recipients died after their initial STCS TX with functioning allograft(s), $45 \%$ of patients with GF died directly after it (figure 2, left panel). Thirty-three SOT recipients died after a consecutive TX, whereas the allograft(s) transplanted there have still functioned.

\section{Rejections and post-transplant ID and cancer}

Allograft rejections were more frequent among lung and heart transplant recipients, and rejection episodes were mostly observed within the first-year post-transplant $(80.6 \%)$. The first rejection occurred significantly earlier after heart transplants (18 vs 76 days post-transplant). Among SOT recipients with GF, around half of patients had a previous rejection episode (292/538, 54.3\%). 


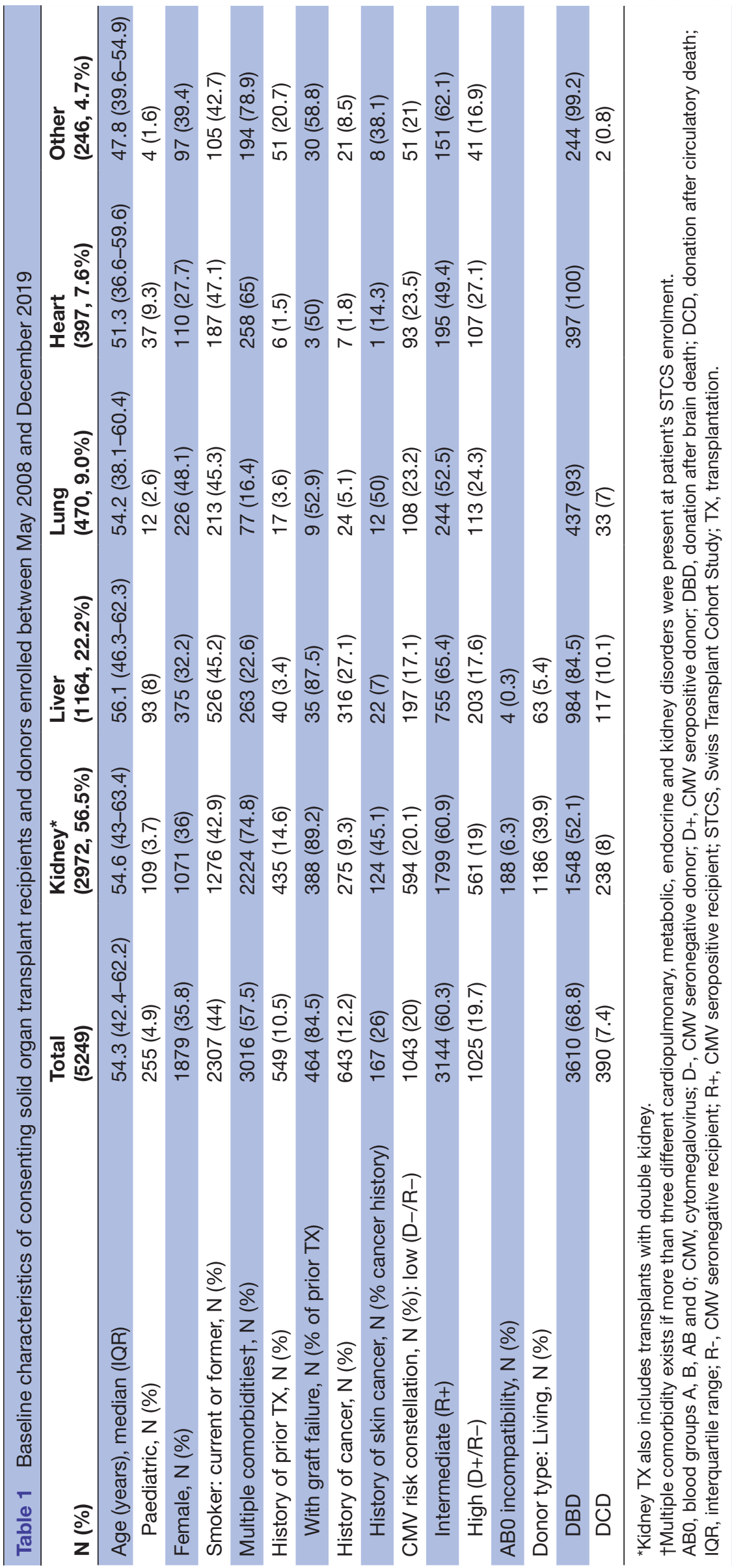




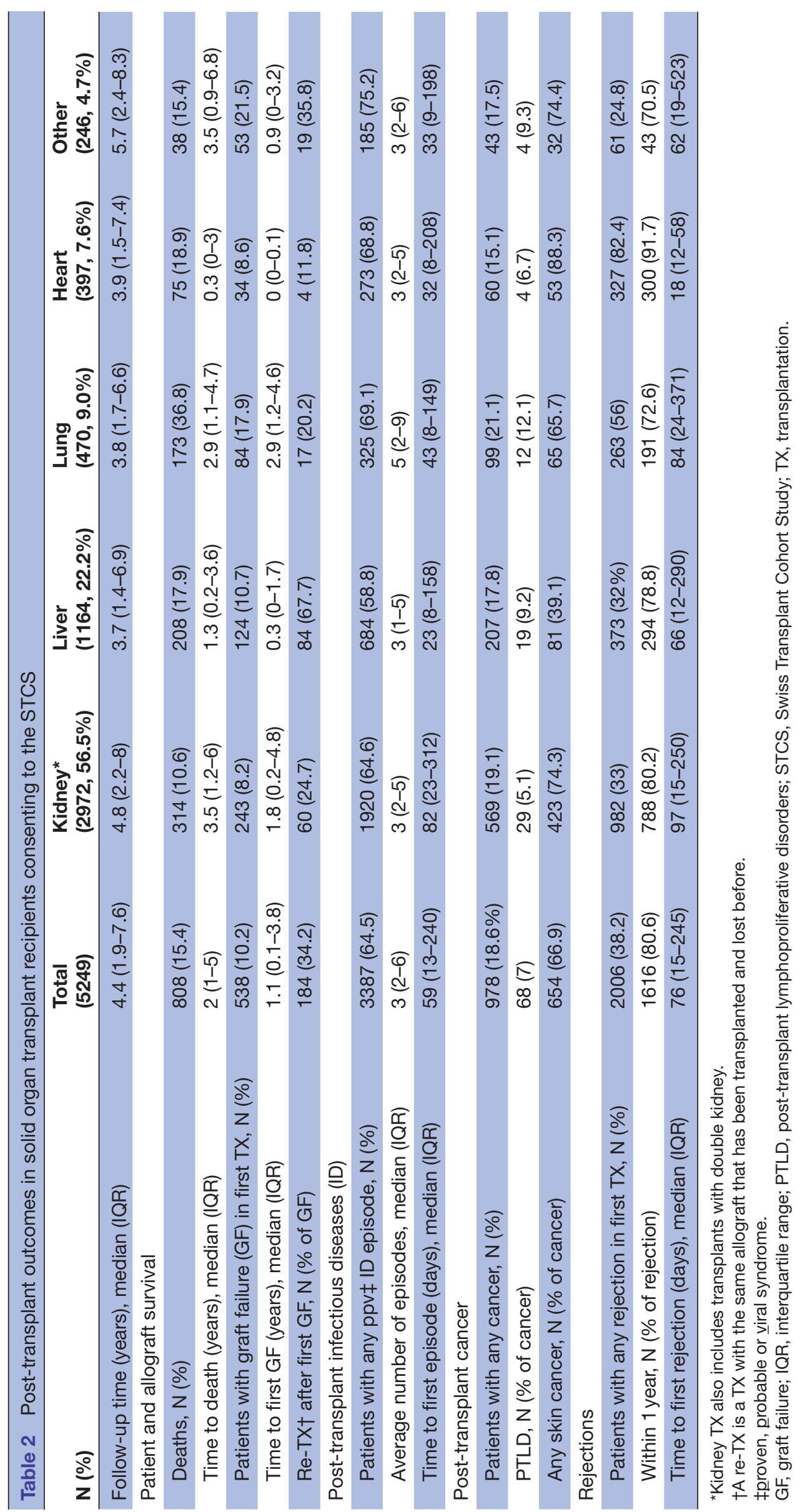




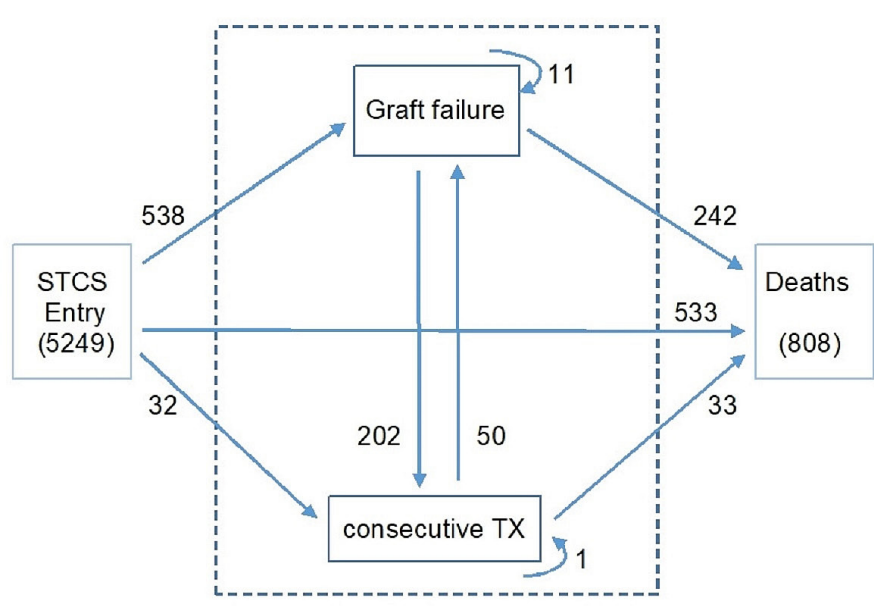

\begin{tabular}{|l|r|}
\hline & N (\%) \\
\hline Allografts transplanted & 5692 \\
\hline Allograft failures (GF) & $599(10.5 \%)$ \\
\hline - single TX & $546(91.2 \%)$ \\
\hline - combined TX & $53(8.8 \%)$ \\
\hline Patients with at least one GF & $543(10.3 \%)$ \\
\hline - multiple GF & $53(9.8 \%)$ \\
\hline - from different TX & $42(79.2 \%)$ \\
\hline - complete GF in combined TX & $11(20.8 \%)$ \\
\hline - re-transplant after GF & $187(34.4 \%)$ \\
\hline Deaths & $808(15.4 \%)$ \\
\hline - with functioning allograft(s) & $536(66.3 \%)$ \\
\hline - following GF & $272(33.7 \%)$ \\
\hline Patients with more than one TX in STCS & $221(4.2 \%)$ \\
\hline - second transplant only & $27(12.2 \%)$ \\
\hline
\end{tabular}

Figure 2 States and transitions after STCS enrolment and information on graft failures, consecutive transplantations and deaths in consenting solid organ transplant recipients during follow-up. ${ }^{1}$ Patients had exclusively TX with organs that had not been transplanted to them before while STCS follow-up. GF, graft failure; STCS, Swiss Transplant Cohort Study; TX, transplantation.

Around two-thirds of consenting SOT recipients experienced at least one proven or probable ID episode or an ID with viral syndrome. The proportion among liver transplant recipients was slightly lower $(58.8 \%)$, and the average number of ID episodes per patient was highest among lung transplant recipients (5 vs 3 ). At least one post-transplant cancer episode was observed in almost one thousand patients, skin cancer was diagnosed in twothirds of these cases whereas the proportion was lower among liver recipients (39.9\%) and higher in heart transplant recipients $(88.3 \%)$. The incidence of PTLD was low across all TX programmes (7\%).

\section{Findings to date}

Transplant infectiuous diseases have been established as a major focus of interest with a track record on various critical issues related to CMV infections. ${ }^{40-46}$ Other areas of interest have emerged, such as airway microbiome assessments in lung transplant recipients, ${ }^{25} 47-49$ T-cell response studies of BK virus infections in kidney transplant recipients, ${ }^{50}$ and association studies of genetic host factors with various ID phenotypes. ${ }^{40} 444551-54$ A landmark study showed that infections still represent a major burden, with rare opportunistic pathogens and a worrisome predominance of bacteria with potential antimicrobial resistance..$^{55}$ The group studied the epidemiology of respiratory viral infections and associated mortality in SOT patients and highlighted the burden of these infections in non-lung recipients as well. ${ }^{56}$ Several publications are the result of international collaborations, such as with the Spanish Network for Research in Infectious Diseases, ${ }^{57-59}$ the European Study Group for Nocardia in solid organ $\mathrm{TX}^{2460}$ and the European Society of Clinical Microbiology and Infectious Diseases parasitology study group on toxoplasmosis in transplant recipients. ${ }^{26}$ The STCS ID group has also contributed first insights in SARSCoV-2 infections among SOT recipients in Switzerland. ${ }^{61}$ The increased involvement of the STCS Immunology working group in recent years led to detailed HLA typing data, especially for kidney transplant recipients. ${ }^{29}$ A casecontrol study evaluated the association between viraemia
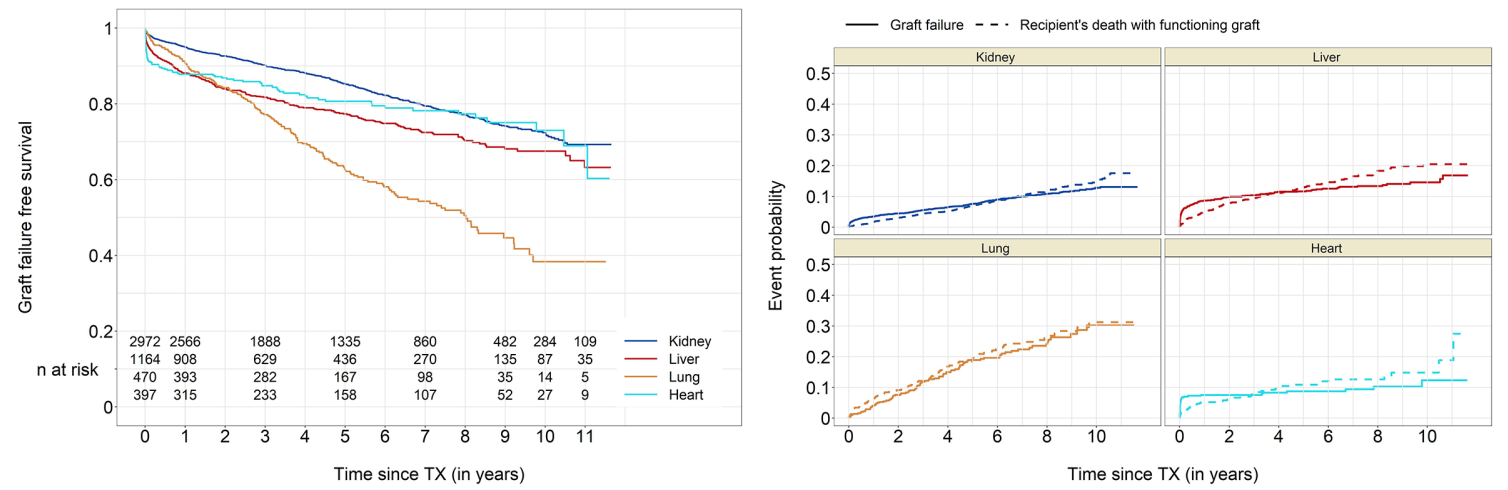

Figure 3 Graft failure free survival (left) and cumulative incidences for graft failure and death despite organ viability (right) in consenting solid organ transplant recipients of the four main TX programmes. Graft failure free survival is defined by the absence of graft failure and patient's death during follow-time. STCS, Swiss Transplant Cohort Study; TX, transplantation. 
and BK polyomavirus specific immunity, ${ }^{28} 50$ and a pilot study revealed the potential of donor-specific memory B cell-derived HLA antibodies as a novel tool to supplement serum HLA antibody analysis for pre-transplant risk stratification in patients with DSA. ${ }^{27}$ Oncology studies surveyed solid and skin cancers among SOT recipients regarding incidence and risk factors. ${ }^{30}{ }^{31}$ Low incidence of PTLD was shown. ${ }^{32}$ In a genome-wide association study, a genetic alteration has been evidenced as a susceptibility factor for squamous cell carcinoma among SOT recipients in Switzerland.$^{36}$ Novel original activities focusing on genetic predictors of metabolic complications, such as new onset post-transplant diabetes ${ }^{334}{ }^{36-64}$ or complement gene polymorphisms associated with acute kidney allograft rejection further underline the potential of the STCS. ${ }^{65}$ An international collaboration with the University of Alberta showed that polymorphisms in the leucocyte immunoglobulin-like receptor subfamily B member 1 influence the control of CMV. ${ }^{35}$ The STCS 'PSIG' presented seminal findings on the evolution of body weight parameters post-transplant ${ }^{6667}$ on the evolution and predictive value of drug adherence, ${ }^{20}$ on workability after TX, ${ }^{68-70}$ and on health-related quality of life posttransplant, as sleep quality ${ }^{19}$ or psychological distress. ${ }^{71}$ The pioneering project GERAS examined frailty and mild cognitive impairment in kidney transplant recipients from biopsychosocial and health-economic perspectives. ${ }^{21}$ The predictive value of the pre-transplant social adaptability index on post-transplant clinical outcomes was investigated in kidney recipients. ${ }^{72}$

\section{FUTURE PERSPECTIVES}

The STCS has been very productive in recent years and contributed valuable findings to the field of TX medicine. In the future, using long-term follow-up data, the STCS will intensify its research efforts in personalised medicine and design patient-tailored, individualised immunosuppression and antimicrobial prophylaxis schemes. The STCS also strives to enter more collaborations with other national and international cohorts, registers or research groups, and to initiate randomised interventional trials applicable in the STCS framework.

A mobile health application ('mTX') will be developed as an interaction platform to connect transplant patients, their involved healthcare providers (HCP) and the STCS, and it aims to foster the patient - HCP interaction and to support personalised patient disease management. As a member of a consortium of four large national cohorts (consisting of the STCS, the Swiss HIV cohort study (SHCS), the Swiss Clinical Quality Management in rheumatic diseases and the Swiss cohort for longitudinally studying SLE (Systemic Lupus Erthematosus)) and university-based institutions, a solution will be implemented for the linkage of individual patient identification numbers from different sources and bring interoperability into the management of confidential patient-identifying information ('IDEAL') ${ }^{73}$ Since 2018 , the STCS has been participating in the SPHN initiative focussing on implementing common semantic and data interoperability standards. It aims to improve data sharing (eg, immunology and imaging data) between the STCS, and the involved hospitals and TX centres. Further, data linkage to federal death and cancer registries is subject of a current feasibility study.

The interdisciplinary STCS 'PSIG' will continue analysing psychosocial and behavioural outcomes over the transplant continuum and invest in innovative e-health supported care models allowing personalised health approaches ('SMILe'). ${ }^{74-76}$ The STCS biobank will participate in the Swiss biobank platform to address standardisation issues also as a prerequisite to ensure interoperability and to improve the findability of samples ('eCatalogue'). It is further involved in the 'BioLink' project where several Swiss cohorts join biobanking efforts to assess pharmacogenomic and metabolomic predispositions for ID predisposition. ${ }^{77}$

Results from the first two randomised interventional trials with patient enrolment completed in late 2020 will be published soon. The first trial aims to adapt the duration of prophylaxis against CMV according to cellmediated anti-CMV immunity, and the second compared immunogenicity, safety and efficacy of three influenza vaccines. The 'Persimune intercohort collaboration project' merging more than 10000 SOT recipients from both the STCS and the MATCH cohort from Denmark evaluates different strategies against CMV infection. ${ }^{78} 79$ A first joint randomised trial on SARS-Cov-2 vaccination in immunosuppressed patients is planned in cooperation with the SHCS. The STCS Genetics working group aims to collaborate with other cohorts or groups of investigators, such as the $i$ GeneTRA $i \mathrm{~N}$, to reinforce the use of genetic data for non-infectious phenotypes.

\section{STRENGTHS AND LIMITATIONS}

The strength of this national cohort lies in its uniqueness in terms of integration of all SOT programmes and extensive data collection within an elaborated framework. It enables a complete assessment of patientspecific, transplant-specific and centre-specific activities in Switzerland and also allows a comprehensive evaluation of patient-reported outcomes. The underlying dynamic IT structure enables to map the complexity of post-transplant patient care and to identify new pathways contributing to the understanding of patient and allograft survival. In addition, the stored biological material fosters to examine novel biomarkers and genetic determinants regarding post-transplant outcome among the population of SOT recipients. A further key advantage is the rigorous longitudinal data that facilitates recording and periodically updating of specific risk profiles to reflect changes in patients' disease- and treatment status. The high consent rate and a low drop-out rate mirrors the acceptance among the cohort participants and has thus steadily improved the quality of the cohort data and study 
results. Due to the legal mandate, there is hardly any missing information in minimal compulsory data from SOT recipients that have been enrolled since May 2008.

Despite all the advantages, the cohort also struggles with some weaknesses, some of which will be addressed in the future. There is a potential lack of some information on post-transplant events of SOT recipients that may have occurred outside TX centres. In addition, the STCS so far only captures a limited amount of donor data and a direct link to waiting list data can be requested but is not automatically provided. So far, the STCS has a fixed biobanking sampling scheme that ensures regular collection of biological material, but makes it impossible to adapt to important events. Further developments such as a personalised sampling or prediction models for future sample usage are planned to support the long-term sustainability of the STCS biobank.

\section{Author affiliations}

${ }^{1}$ Clinic for Transplantation Immunology and Nephrology, University Hospital Basel, Basel, Switzerland

${ }^{2}$ Division of Infectious Diseases and Hospital Epidemiology, University Hospital Zurich, Zurich, Switzerland

${ }^{3}$ Transplant Infectious Diseases Unit, University Hospitals Geneva and Faculty of Medicine, Geneva, Switzerland

${ }^{4}$ Transplantation Center, Lausanne University Hospital and University of Lausanne, Lausanne, Switzerland

${ }^{5}$ Service of Infectious Diseases, Lausanne University Hospital and University of Lausanne, Lausanne, Switzerland

${ }^{6}$ Department for Visceral Surgery and Medicine, Inselspital, University Hospital Bern and Bern University, Bern, Switzerland

${ }^{7}$ Nephrology and Transplantation Medicine, Kantonsspital St Gallen, Sankt Gallen,

Switzerland

${ }^{8}$ Department of Public Health, Institute of Nursing Science, University of Basel, Basel, Switzerland

${ }^{9}$ Center for Laboratory Medicine, University Institute of Clinical Chemistry, Inselspital University Hospital Bern, Bern, Switzerland

\section{Twitter Sabina De Geest @SABINADG and Alexander Leichtle @Leichtle}

Collaborators The members of the Swiss Transplant Cohort Study are: Patrizia Amico, Andres Axel, John-David Aubert, Vanessa Banz, Beckmann Sonja, Guido Beldi, Christoph Berger, Ekaterine Berishvili, Annalisa Berzigotti, Isabelle Binet, Pierre-Yves Bochud, Sanda Branca, Heiner Bucher, Thierry Carrel, Emmanuelle Catana, Yves Chalandon, Sabina De Geest, Olivier De Rougemont, Sophie De Seigneux, Michael Dickenmann, Joëlle Lynn Dreifuss, Michel Duchosal, Thomas Fehr, Sylvie Ferrari-Lacraz, Nicola Franscini, Christian Garzoni, Paola Gasche Soccal, Christophe Gaudet, Déla Golshayan, Nicolas Goossens, Karine Hadaya, Jörg Halter, Dominik Heim, Christoph Hess, Sven Hillinger, Hans Hirsch, Patricia Hirt, Günther Hofbauer, Uyen Huynh-Do, Franz Immer, Michael Koller, Mirjam Laager, Bettina Laesser, Roger Lehmann, Alexander Leichtle, Christian Lovis, Oriol Manuel, Hans-Peter Marti, Pierre Yves Martin, Michele Martinelli, Valérie McLin, Katell Mellac, Aurélia Merçay, Karin Mettler, Nicolas Mueller, Antonia Müller, Ulrike Müller-Arndt, Beat Müllhaupt, Mirjam Nägeli, Graziano Oldani, Manuel Pascual, Klara Posfay-Barbe, Juliane Rick, Anne Rosselet, Simona Rossi, Silvia Rothlin, Frank Ruschitzka, Thomas Schachtner, Urs Schanz, Stefan Schaub, Aurelia Schnyder, Macé Schuurmans, Thierry Sengstag, Federico Simonetta, Susanne Stampf, Jürg Steiger, Guido Stirniman, Ueli Stürzinger, Christian Van Delden, Jean-Pierre Venetz, Jean Villard, Julien Vionnet, Madeleine Wick, Markus Wilhlem, Patrick Yerly.

Contributors Guarantor: MK; Conceptualisation, writing: SSt, MK; Formal analysis: SSt; Review, edit, revision: NJM, OM, MP, CVD, IB, SDG, VBW, ABL, P-YB, SSc and JS. All authors have read and agreed to the published version of the manuscript.

Funding The Swiss Transplant Cohort Study (STCS) is funded by the Swiss National Science Foundation (SNSF) since the beginning (grant number: 33CS30_177522/2). Universitäre Medizin Schweiz supports the STCS and its quality of care monitoring.
Competing interests None declared.

Patient and public involvement Patients and/or the public were not involved in the design, or conduct, or reporting, or dissemination plans of this research.

Patient consent for publication Not applicable.

Ethics approval Competent ethics committee of each transplant centre approved the STCS (EKNZ project number 2018-02394).

Provenance and peer review Not commissioned; externally peer reviewed.

Data availability statement Data are available on reasonable request. STCS data are open for researchers and the data access is regulated by the STCS scientifc committee.

Open access This is an open access article distributed in accordance with the Creative Commons Attribution Non Commercial (CC BY-NC 4.0) license, which permits others to distribute, remix, adapt, build upon this work non-commercially, and license their derivative works on different terms, provided the original work is properly cited, appropriate credit is given, any changes made indicated, and the use is non-commercial. See: http://creativecommons.org/licenses/by-nc/4.0/.

\section{ORCID iDs}

Susanne Stampf http://orcid.org/0000-0003-3399-5078

Oriol Manuel http://orcid.org/0000-0001-7607-0943

Alexander Leichtle http://orcid.org/0000-0002-6528-9904

\section{REFERENCES}

1 Schaub S, Immer F, Steiger J. Organ transplantation in Switzerland. Transplantation 2019;103:853-6.

2 Federal act on the transplantation of organs, tissues and cells, 2007. Available: https://www.admin.ch/opc/en/classified-compilation/ 20010918/index.html [Accessed 1 Jan 2020].

3 Koller MT, van Delden C, Müller NJ, et al. Design and methodology of the Swiss transplant cohort study (STCS): a comprehensive prospective nationwide long-term follow-up cohort. Eur J Epidemiol 2013:28:347-55.

4 Koller Met al. Die Schweizerische Transplantationskohortenstudie (STCS). Schweizerische Ärztezeitschrift 2009;90:953-5.

5 Branca-Dragan S. Swiss Transplant Cohort Study Report (May 2008 December 2019. Basel: University Hospital Basel, 2020.

6 Weiss J, Kocher M, Immer F. International collaboration and organ exchange in Switzerland. J Thorac Dis 2014;7:543-8.

7 Immer F. Rechtliche Aspekte Der Organzuteilung - Licht und Schatten. Schweizerische Ärztezeitschrift 2015;96:1780-2.

8 Immer F. Aktuelle Entwicklungen auf dem Gebiet Der Organspende und transplantation in Der Schweiz. Anaesth J 2017;2:24-7.

9 Weiss J, Beyeler F, Immer FF, et al. Heart allocation and transplantation in Switzerland since the introduction of the Swiss organ allocation system (SOAS). Swiss Med Wkly 2014;144:w14057.

10 Hadaya K, Fehr T, Rüsi B, et al. Kidney paired donation: a plea for a Swiss National programme. Swiss Med Wkly 2015;145:w14083.

11 Mierzejewska B, Durlik M, Lisik W, et al. Current approaches in national kidney paired donation programs. Ann Transplant 2013;18:112-24.

12 Verordnung über das nationale Überkreuz-Lebendspende-Programm (Überkreuz-Lebendspende-Verordnung), 2017. Available: https:// www.bag.admin.ch/bag/de/home/medizin-und-forschung/transpla ntationsmedizintransplantationsmedizin/rechtsetzungsprojekterec htsetzungsprojekte-in-der-transplantationsmedizintransplantationsm edizin/ueber-kreuz-lebendspende-verordnung.html

13 Thiel GT, Nolte C, Tsinalis D. Prospective Swiss cohort study of living-kidney donors: study protocol. BMJ Open 2011;1:e000202.

14 Burkhalter F, Huynh-Do U, Hadaya K, et al. Early complications after living donor nephrectomy: analysis of the Swiss Organ Living Donor Health Registry. Swiss Med Wkly 2017;147:w14497.

15 Magiorakos A-P, Srinivasan A, Carey RB, et al. Multidrug-Resistant, extensively drug-resistant and pandrug-resistant bacteria: an international expert proposal for interim standard definitions for acquired resistance. Clin Microbiol Infect 2012;18:268-81.

16 Humar A, Michaels M, AST ID Working Group on Infectious Disease Monitoring. American Society of transplantation recommendations for screening, monitoring and reporting of infectious complications in immunosuppression trials in recipients of organ transplantation. $\mathrm{Am} \mathrm{J}$ Transplant 2006;6:262-74.

17 Roufosse C, Simmonds N, Clahsen-van Groningen M, et al. A 2018 reference guide to the Banff classification of renal allograft pathology. Transplantation 2018;102:1795-814. 
18 Ponikowski P, Voors AA, Anker SD, et al. 2016 ESC guidelines for the diagnosis and treatment of acute and chronic heart failure. Eur Heart $J$ 2016;37:2129-200.

19 Burkhalter H, Denhaerynck K, Huynh-Do U, et al. Change of sleep quality from pre- to 3 years post-solid organ transplantation: the Swiss transplant cohort study. PLoS One 2017;12:e0185036.

20 De Geest S, Burkhalter H, Bogert L, et al. Describing the evolution of medication nonadherence from pretransplant until 3 years post-transplant and determining pretransplant medication nonadherence as risk factor for post-transplant nonadherence to immunosuppressives: the Swiss transplant cohort study. Transpl Int 2014;27:657-66.

21 Mauthner O, Claes V, Walston J, et al. Exploring frailty and mild cognitive impairmEnt in kidney tRansplantation to predict biomedicAl, psychosocial and health cost outcomeS (GERAS): protocol of a nationwide prospective cohort study. J Adv Nurs 2017:73:716-34.

22 De Geest S, Burkhalter H, Berben L, et al. The Swiss transplant cohort study's framework for assessing lifelong psychosocial factors in solid organ transplants. Prog Transplant 2013;23:235-46.

23 Lorent M, Giral M, Pascual M, et al. Mortality prediction after the first year of kidney transplantation: an observational study on two European cohorts. PLoS One 2016;11:e0155278.

24 Coussement J, Lebeaux D, van Delden C, et al. Nocardia infection in solid organ transplant recipients: a multicenter European casecontrol study. Clin Infect Dis 2016;63:338-45.

25 Pison C, Magnan A, Botturi K, et al. Prediction of chronic lung allograft dysfunction: a systems medicine challenge. Eur Respir $J$ 2014;43:689-93.

26 Robert-Gangneux F, Meroni V, Dupont D, et al. Toxoplasmosis in transplant recipients, Europe, 2010-2014. Emerg Infect Dis 2018;24:1497-504.

27 Wehmeier C, Karahan GE, Krop J, et al. Donor-Specific B cell memory in Alloimmunized kidney transplant recipients: first clinical application of a novel method. Transplantation 2020;104:1026-32.

28 Willhelm M, Wilk S, Kaur A, et al. Can HLA-B51 protect against BKPyV-DNAemia? Transplantation 2019;103:e384-5.

29 Krisl A, Stampf S, Hauri D, et al. Immunosuppression management in renal transplant recipients with normal-immunological risk: 10-year results from the Swiss transplant cohort study. Swiss Med Wkly 2020;150:w20354.

30 Lengwiler E, Stampf S, Zippelius A, et al. Solid cancer development in solid organ transplant recipients within the Swiss transplant cohort study. Swiss Med Wkly 2019;149:w20078.

31 Stenz NA, Stampf S, Arnold AW, et al. Skin cancer development in solid organ transplant recipients in Switzerland (Swiss transplant cohort study). Dermatology 2021;237:970-80.

32 Steiner R, Kridel R, Giostra E, et al. Low 5-year cumulative incidence of post-transplant lymphoproliferative disorders after solid organ transplantation in Switzerland. Swiss Med Wkly 2018;148:w14596.

33 Quteineh L, Wójtowicz A, Bochud P-Y, et al. Genetic immune and inflammatory markers associated with diabetes in solid organ transplant recipients. Am J Transplant 2019;19:238-46.

34 Saigi-Morgui N, Quteineh L, Bochud P-Y, et al. Weighted genetic risk scores and prediction of weight gain in solid organ transplant populations. PLoS One 2016;11:e0164443.

35 Yu K, Davidson CL, Wójtowicz A, et al. Lilrb1 polymorphisms influence posttransplant HCMV susceptibility and ligand interactions. J Clin Invest 2018:128:1523-37.

36 Kuzmanov A, Qi W, Stenz N, et al. rs34567942 a novel susceptibility single nucleotide polymorphism for cutaneous squamous cell carcinoma in organ transplant recipients. Acta Derm Venereol 2019;99:1303-4.

37 Berger C, Bochud P-Y, Boggian K, et al. The swiss transplant cohort study: lessons from the first 6 years. Curr Infect Dis Rep 2015; $17: 486$

38 Swiss Biobanking Platform. 2019 Annual report, 2019. Available: https://swissbiobanking.ch

39 Mulley WR, Kanellis J. Understanding crossmatch testing in organ transplantation: a case-based guide for the general nephrologist. Nephrology 2011;16:125-33.

40 Han SH, Kumar D, Ferreira VH, et al. Human microRNA responses predict cytomegalovirus replication following solid organ transplantation. J Infect Dis 2017;215:537-46.

41 Martin-Gandul C, Stampf S, Héquet D, et al. Preventive strategies against cytomegalovirus and incidence of $\alpha$-Herpesvirus infections in solid organ transplant recipients: a nationwide cohort study. $A m \mathrm{~J}$ Transplant 2017:17:1813-22.

42 Tschan-Plessl A, Stern M, Schmied L, et al. Human cytomegalovirus infection enhances NK cell activity in vitro. Transplant Direct 2016;2:e89.
43 Stern M, Hirsch H, Cusini A, et al. Cytomegalovirus serology and replication remain associated with solid organ graft rejection and graft loss in the era of prophylactic treatment. Transplantation 2014;98:1013-8.

44 Manuel O, Wójtowicz A, Bibert S, et al. Influence of IFNL3/4 polymorphisms on the incidence of cytomegalovirus infection after solid organ transplantation. J Infect Dis 2015;211:906-14.

45 Gonzalez A, Schmitter K, Hirsch HH, et al. KIR-associated protection from CMV replication requires pre-existing immunity: a prospective study in solid organ transplant recipients. Genes Immun 2014;15:495-9.

46 Manuel O, Kralidis G, Mueller NJ, et al. Impact of antiviral preventive strategies on the incidence and outcomes of cytomegalovirus disease in solid organ transplant recipients. Am J Transplant 2013;13:2402-10.

47 Beaume M, Köhler T, Greub G, et al. Rapid adaptation drives invasion of airway donor microbiota by Pseudomonas after lung transplantation. Sci Rep 2017;7:40309.

48 Beaume M, Lazarevic V, Köhler T, et al. Microbial communities of conducting and respiratory zones of Lung-Transplanted patients. Front Microbiol 2016;7:1749.

49 Bernasconi E, Pattaroni C, Koutsokera A, et al. Airway microbiota determines innate cell inflammatory or tissue remodeling profiles in lung transplantation. Am J Respir Crit Care Med 2016;194:1252-63.

50 Leboeuf C, Wilk S, Achermann R, et al. Bk Polyomavirus-Specific 9mer CD8 T cell responses correlate with clearance of BK viremia in kidney transplant recipients: first report from the Swiss transplant cohort study. Am J Transplant 2017;17:2591-600.

51 Wójtowicz A, Lecompte TD, Bibert S, et al. Ptx3 polymorphisms and invasive mold infections after solid organ transplant. Clin Infect Dis 2015;61:619-22.

52 Wójtowicz A, Gresnigt MS, Lecompte T, et al. II1B and DEFB1 polymorphisms increase susceptibility to invasive mold infection after solid organ transplantation. J Infect Dis 2015;211:1646-57.

53 Schmied L, Terszowski G, Gonzalez A, et al. Protection from varicella zoster in solid organ transplant recipients carrying killer cell immunoglobulin-like receptor B haplotypes. Transplantation 2015;99:2651-5

54 Buhler S, Giostra E, Gbame C, et al. A significant effect of the killer cell immunoglobulin-like receptor ligand human leucocyte antigen-C on fibrosis progression in chronic $\mathrm{C}$ hepatitis with or without liver transplantation. Liver Int 2016;36:1331-9.

55 van Delden C, Stampf S, Hirsch HH, et al. Burden and Timeline of infectious diseases in the first year after solid organ transplantation in the Swiss transplant cohort study. Clin Infect Dis 2020;71:e159-69.

56 Mombelli M, Lang BM, Neofytos D, et al. Burden, epidemiology, and outcomes of microbiologically confirmed respiratory viral infections in solid organ transplant recipients: a nationwide, multi-season prospective cohort study. Am J Transplant 2021;21:1789-800.

57 López-Medrano F, Fernández-Ruiz M, Silva JT, et al. Clinical presentation and determinants of mortality of invasive pulmonary aspergillosis in kidney transplant recipients: a multinational cohort study. Am J Transplant 2016;16:3220-34.

58 López-Medrano F, Silva JT, Fernández-Ruiz M, et al. Risk factors associated with early invasive pulmonary aspergillosis in kidney transplant recipients: results from a multinational matched casecontrol study. Am J Transplant 2016;16:2148-57.

59 López-Medrano F, Fernández-Ruiz M, Silva JT, et al. Multinational case-control study of risk factors for the development of late invasive pulmonary aspergillosis following kidney transplantation. Clin Microbiol Infect 2018;24:192-8.

60 Lebeaux D, Freund R, van Delden C, et al. Outcome and treatment of nocardiosis after solid organ transplantation: new insights from a European study. Clin Infect Dis 2017;64:1396-405.

61 Tschopp J, L'Huillier AG, Mombelli M, et al. First experience of SARS-CoV-2 infections in solid organ transplant recipients in the Swiss transplant cohort study. Am J Transplant 2020;20:2876-82.

62 Quteineh L, Bochud P-Y, Golshayan D, et al. Crtc2 polymorphism as a risk factor for the incidence of metabolic syndrome in patients with solid organ transplantation. Pharmacogenomics J 2017;17:69-75

63 Métrich M, Mehmeti F, Feliciano H, et al. Adrenergic receptor polymorphism and maximal exercise capacity after orthotopic heart transplantation. PLoS One 2016;11:e0163475.

64 Saigi-Morgui N, Quteineh L, Bochud P-Y, et al. Genetic and clinic predictors of new onset diabetes mellitus after transplantation. Pharmacogenomics J 2019;19:53-64.

65 Golshayan D, Wójtowicz A, Bibert S, et al. Polymorphisms in the lectin pathway of complement activation influence the incidence of acute rejection and graft outcome after kidney transplantation. Kidney Int 2016;89:927-38. 
66 Beckmann S, Nikolic N, Denhaerynck K, et al. Evolution of body weight parameters up to 3 years after solid organ transplantation: The prospective Swiss Transplant Cohort Study. Clin Transplant 2017;31. doi:10.1111/ctr.12896. [Epub ahead of print: 24 Jan 2017].

67 Beckmann S, Denhaerynck K, Stampf S, et al. New-Onset obesity after liver transplantation-outcomes and risk factors: the Swiss transplant cohort study. Transpl Int 2018;31:1254-67.

68 Danuser B, Simcox A, Studer R, et al. Employment 12 months after kidney transplantation: an in-depth bio-psycho-social analysis of the Swiss transplant cohort. PLoS One 2017;12:e0175161.

69 Vieux L, Simcox AA, Mediouni Z, et al. Predictors of return to work 12 months after solid organ transplantation: results from the Swiss transplant cohort study. J Occup Rehabil 2019;29:462-71.

70 Branca-Dragan S, Koller MT, Danuser B, et al. Evolution of disability pension after renal transplantation: methods and results of a database linkage study of the Swiss transplant cohort study and Swiss disability insurance. Swiss Med Wkly 2021;151:w30027.

71 Bleisch B, Schuurmans MM, Klaghofer R, et al. Health-Related quality of life and stress-related post-transplant trajectories of lung transplant recipients: a three-year follow-up of the Swiss transplant cohort study. Swiss Med Wkly 2019;149:w20019.

72 Denhaerynck K, Goldfarb-Rumyantzev AS, Sandhu G, et al. PreTransplant social adaptability index and clinical outcomes in renal transplantation: the Swiss transplant cohort study. Clin Transplant 2021;35:e14218.
73 A Swiss national IT infrastructure project to improve data exchange between clinics and research institutions, 2021. Available: https:// ideal-project.ch/

74 Leppla L, Mielke J, Kunze M, et al. Clinicians and patients perspectives on follow-up care and eHealth support after allogeneic hematopoietic stem cell transplantation: a mixed-methods contextual analysis as part of the SMILE study. Eur J Oncol Nurs 2020;45:101723.

75 Ribaut J, Leppla L, Teynor A, et al. Theory-driven development of a medication adherence intervention delivered by eHealth and transplant team in allogeneic stem cell transplantation: the SMILE implementation science project. BMC Health Serv Res 2020;20:827.

76 Leppla L, Hobelsberger S, Rockstein D, et al. Implementation science meets software development to create eHealth components for an integrated care model for allogeneic stem cell transplantation facilitated by eHealth: the SMILE study as an example. J Nurs Scholarsh 2021;53:35-45.

77 Swiss Biobank platform. Project BioLink, 2020. Available: https:// swissbiobanking.ch/biolink/

78 Wareham NE, Mocroft A, Sengeløv H, et al. The value of EBV DNA in early detection of post-transplant lymphoproliferative disorders among solid organ and hematopoietic stem cell transplant recipients. J Cancer Res Clin Oncol 2018;144:1569-80.

79 Ekenberg C, da Cunha-Bang C, Lodding IP, et al. Evaluation of an electronic, patient-focused management system aimed at preventing cytomegalovirus disease following solid organ transplantation. Transpl Infect Dis 2020;22:e13252. 